\title{
Video Article \\ Clinical-oriented Three-dimensional Gait Analysis Method for Evaluating Gait Disorder
}

\author{
Masahiko Mukaino ${ }^{1}$, Kei Ohtsuka ${ }^{2}$, Hiroki Tanikawa ${ }^{2}$, Fumihiro Matsuda ${ }^{2}$, Junya Yamada ${ }^{3}$, Norihide Itoh ${ }^{4}$, Eiichi Saitoh ${ }^{1}$ \\ ${ }^{1}$ Department of Rehabilitation Medicine, School of Medicine, Fujita Health University \\ ${ }^{2}$ Faculty of Rehabilitation, School of Health Science, Fujita Health University \\ ${ }^{3}$ Department of Rehabilitation, Fujita Health University Hospital \\ ${ }^{4}$ Department of Advanced Rehabilitation Medicine, Graduate School of Medical Science, Kyoto Prefectural University of Medicine
}

Correspondence to: Masahiko Mukaino at masahikovn@gmail.com

URL: https://www.jove.com/video/57063

DOI: doi: $10.3791 / 57063$

Keywords: Behavior, Issue 133, three-dimensional gait analysis, gait disorder, stroke, rehabilitation, treadmill, clinical evaluation

Date Published: 3/4/2018

Citation: Mukaino, M., Ohtsuka, K., Tanikawa, H., Matsuda, F., Yamada, J., Itoh, N., Saitoh, E. Clinical-oriented Three-dimensional Gait Analysis Method for Evaluating Gait Disorder. J. Vis. Exp. (133), e57063, doi:10.3791/57063 (2018).

\section{Abstract}

Three-dimensional gait analysis (3DGA) is shown to be a useful clinical tool for the evaluation of gait abnormality due to movement disorders. However, the use of 3DGA in actual clinics remains uncommon. Possible reasons could include the time-consuming measurement process and difficulties in understanding measurement results, which are often presented using a large number of graphs. Here we present a clinician-friendly 3DGA method developed to facilitate the clinical use of 3DGA. This method consists of simplified preparation and measurement processes that can be performed in a short time period in clinical settings and intuitive results presentation to facilitate clinicians' understanding of results. The quick, simplified measurement procedure is achieved by the use of minimum markers and measurement of patients on a treadmill. To facilitate clinician understanding, results are presented in figures based on the clinicians' perspective. A Lissajous overview picture (LOP), which shows the trajectories of all markers from a holistic viewpoint, is used to facilitate intuitive understanding of gait patterns. Abnormal gait pattern indices, which are based on clinicians' perspectives in gait evaluation and standardized using the data of healthy subjects, are used to evaluate the extent of typical abnormal gait patterns in stroke patients. A graph depicting the analysis of the toe clearance strategy, which depicts how patients rely on normal and compensatory strategies to achieve toe clearance, is also presented. These methods could facilitate implementation of 3DGA in clinical settings and further encourage development of measurement strategies from the clinician's point of view.

\section{Video Link}

The video component of this article can be found at https://www.jove.com/video/57063/

\section{Introduction}

Previous studies have shown the usefulness of three-dimensional gait analysis (3DGA) for the evaluation of gait after stroke ${ }^{1,2,3}$. Studies with high-quality motion analysis systems have provided substantial insight into human gait patterns, not only of those of healthy subjects but also those due to various movement disorders such as stroke or cerebral palsy ${ }^{4,5}$. Understanding pathology, assessing patients prior to treatment for planning, or monitoring intervention effects could all be promoted with $3 D G A^{6}$. Further, several recent studies have demonstrated the potential of 3DGA to provide a guide for rehabilitative training ${ }^{7,8}$.

However, the use of 3DGA in daily clinical practice is still limited. One of the major issues is its time-consuming process. Marker sets commonly used in gait analysis ${ }^{9,10,11}$ consist of more than 30 markers for whole-body measurement. These marker sets enable very precise estimation of limb and trunk movement. This contributes to accuracy of the analysis, which is usually done from the data of limited number of steps that could be captured by cameras placed around a walkway. However, this requires a time-consuming preparation and measurement processes, which impedes the use of 3DGA in daily clinical practice.

Another drawback to using 3DGA in a clinical setting is that it can be difficult to interpret the results provided ${ }^{12}$. The results of 3DGA are commonly shown in graphs depicting parameters such as joint angles and displacement of body parts. However, gait evaluation in the rehabilitation clinics not only involves evaluating the movement of body parts but also holistic movement patterns. The latter can only be understood by assessing the relationships between those parameters, and the difficulty involved in doing so makes clinicians less willing to use 3DGA.

To address these problems and facilitate the use of 3DGA in the rehabilitation clinic, we propose a simplified and intuitive measurement method for 3DGA. The measurement method consists of the following: (1) a simplified marker set with 12 markers; (2) measurement of patients on the treadmill; (3) an intuitive holistic figure of gait patterns; (4) abnormal gait pattern indices validated through clinical observation; and (5) visualization of the features of gait strategy. The protocol shown in this study follows the guidelines of Medical Ethics Committee of the institution. 


\section{Preparation}

NOTE: The measurement method proposed herein utilizes a simplified marker set consisting of twelve markers, as outlined below. The entire measurement process, including preparation, can be performed within $20 \mathrm{~min}$. The feasibility of this protocol in real clinical settings has been verified in previous studies ${ }^{13,14,15,16,17}$.

1. Perform the calibration process according to manufacturer's protocol.

2. Instruct patients to wear easy fit, skintight legwear, and place the colored markers on the patient, as outlined below.

1. Place markers $(30 \mathrm{~mm}$ in diameter) at the following points on the patient's body: both acromia, hips (at points one-third of the way from the greater trochanter of the femur along a line joining each anterior superior iliac spine with each greater trochanter), knees (on the midline of the anterior-posterior diameter of each lateral epicondyle of the femur), ankles (lateral malleoli), toes (fifth metatarsal heads), and iliac crests (at the position of each iliac crest on a vertical line passing through the hips).

\section{Measurement}

1. Set the speed of treadmill and measure gait patterns.

1. Instruct the patient to perform the $10 \mathrm{~m}$ walk test.

2. Set a subjectively comfortable gait speed. Set the treadmill speed, starting at $70 \%$ of the over ground gait and gradually increasing the speed, to find the comfortable gait speed on the treadmill.

3. Measure walking. For a single session it takes $20 \mathrm{~s}$. If the different conditions (e.g. gait speed, orthosis etc.) need to be compared perform several sessions. In that case, have patients rest for 1 minute between the sessions.

NOTE: Although 3DGA systems with infrared cameras are commonly used in 3DGA research, a video-based simplified motion analysis system (sampling frequency: $60 \mathrm{~Hz}$; e.g., Kinematracer) is used in this protocol to shorten the calibration process.

\section{Data Analysis}

\section{Time-distance Factors}

NOTE: Time-distance parameters are common and basic parameters used in gait analysis studies. Events of foot initial contact and foot-off are determined automatically by the system based on toe and ankle marker trajectories.

1. To avoid errors in step detection, have two experienced physical therapists verify the accuracy of timing and adjust it if there are errors in step timing.

2. From these detected step timings, calculate the following:

3. Calculate Cadence (steps $/ \mathrm{min})=60 \times 2 / \mathrm{SC}(\mathrm{s})$

4. Calculate Stride length $(\mathrm{m})=\mathrm{GS}(\mathrm{m} / \mathrm{s}) \times \mathrm{SC}(\mathrm{s})+($ The displacement of ankle marker from foot contact to foot contact of the same side)

5. Calculate Step length $(\mathrm{m})=\mathrm{GS}(\mathrm{m} / \mathrm{s}) \times($ The time from foot contact of one side to foot contact of the other side) $+($ The average difference in the ankle position at foot contact of one side and the other side)

6. Calculate Step width $(m)=$ The average lateral distance between both ankle markers during double stance phase

7. Calculate Duration of double stance: The sum of double stance phase that appears twice during a gait cycle; the time between foot contact of one side and subsequent foot off on the other side.

8. Calculate Duration of single stance: The average time between foot off and subsequent foot contact on the opposite side. NOTE: SC (Step cycle): The average time from heel contact of one side to heel contact of the same side. ${ }^{* *} \mathrm{GS}$ (Gait speed)

2. Lissajous Overview Picture (LOP)

NOTE: LOPs are designed as a figure consisting of marker joint trajectories on major joints with the aim of facilitating understanding rehabilitation patients' holistic gait patterns ${ }^{14}$ (see Figure 1).

1. Generate LOPs from trajectories at the coordinates of ten markers and the virtual center of gravity (COG) in the horizontal (x-y), sagittal $(y-z)$, and coronal (z-x) planes (x: left/right, $y$ : anterior/posterior, $z$ : superior/inferior).

1. Draw the LOP with the software or by constructing a scatterplot that covers the motion range of all the markers on a spreadsheet software. Calculate the virtual COG by hypothesizing each body segment to be as follows: trunk, 0.66; thigh, 0.1; lower thigh, 0.05 ; and foot, 0.02 . Subsequently, calculate COG as the composition center of the segments.

2. At each marker, extract raw data for the three components $(x, y$, and $z)$ for each gait cycle, normalize these by gait cycle, and average the values. Set mean values of the $x$ - and $y$-components of the virtual COG to 0 and use these as references for the $x$ - and $y$ components of the markers.

3. Calculation of Abnormal Gait Index for Hemiparetic Gait

NOTE: Several common gait patterns are known to be features of hemiparetic gait after stroke. These include hip hiking, circumduction, and lateral shift of the trunk ${ }^{18}$. Indices for abnormal gait patterns have been developed to quantify the extent of these gait patterns ${ }^{13,16,17}$. To date, ten abnormal gait indices (hip hiking, circumduction, forefoot contact, retropulsion of the hip, excessive hip external rotation, excessive lateral shift of the trunk over the unaffected side, knee extensor thrust, flexed-knee gait, insufficient knee flexion during the swing phase and medial whip) have been reported. The formula for each index is shown in Table 1.

1. Calculate the raw value of the indices according to the formulas. For example, calculate the raw value of the index for hip hiking as the difference between the maximum value of the z-coordinate of the hip joint marker during the swing phase and the concurrent $z-$ 
coordinate of the contralateral hip joint marker, corrected for the mean left-right difference of the z-coordinate during the double support phase.

2. Calculate the deviation score (t-score) based on measurement data of healthy subjects. Calculate the standardized score as follows: $T$ $=50+10 \times(X-\mu) / \delta(T:$ deviation score; X: individual data; $\mu$ : mean raw value of the healthy subjects; $\delta$ : standard deviation $)$.

\section{Analysis of Toe Clearance Strategy}

NOTE: Strategies for toe clearance during swing vary greatly between healthy subjects and patients with lower limb paresis. In healthy subjects, toe clearance is achieved by limb shortening, with minimal movements of the pelvis or trunk. On the other hand, patients with paresis cannot shorten their limbs to the same extent. For these patients, the resulting compensatory strategies such as pelvic tilt or circumduction also affect toe clearance ${ }^{19}$. In this analysis, the height of toe clearance at mid-swing is divided into two parts: the vertical gain achieved by limb shortening and that gained by compensatory movements, which directly affect toe clearance. The components that comprise toe clearance (vertical component of limb shortening, pelvic obliquity, hip abduction, and vaulting) are calculated as outlined below.

1. Calculate total toe clearance from the $z$-coordinate of the fifth metatarsal head as an index of toe clearance.

2. Calculate the vertical component of limb shortening as the $z$-coordinate of the change in the distance between the hip and the fifth metatarsal head.

3. Calculate the vertical component of compensatory movement by summing three subcomponents, as follows.

1. Calculate the vertical component of pelvic obliquity as the difference in height (z-coordinate) of hip markers.

2. Calculate the vertical component of hip abduction as the distance between the hip and the fifth metatarsal head and the abduction angles. This reflects the upward distance that the foot would have moved as a result of hip abduction if there had been no change in lower limb length.

3. Calculate the vertical component of vaulting from the change in z-coordinate of the contralateral hip between mid-stance and mid-swing ${ }^{20}$.

\section{Representative Results}

The method presented herein is used to evaluate patients with gait disorders. The results of the analysis are presented in Figure 2, Figure 3, and Figure 4. These figures show the results of the simplified 3DGA of a patient with hemiparesis and a healthy control. Figure 2 shows a representative LOP of a stroke patient's complete gait pattern. In this patient, typical gait patterns such as circumduction, hip elevation, and trunk lateral movement were observed. Figure $\mathbf{3}$ is a radar chart showing stroke patients' abnormal gait index (deviation score). Standardized scores of circumduction and hip elevation were high, indicating that those movements in stroke patients were much greater than the standard of healthy subjects. Finally, Figure $\mathbf{4}$ is a graph depicting toe clearance strategies of a stroke patient and an age-matched healthy subject. In the healthy subject, toe clearance is usually achieved by limb shortening, whereas in the stroke patient, it is achieved mainly by compensatory movements such as pelvic obliquity and vaulting.

A

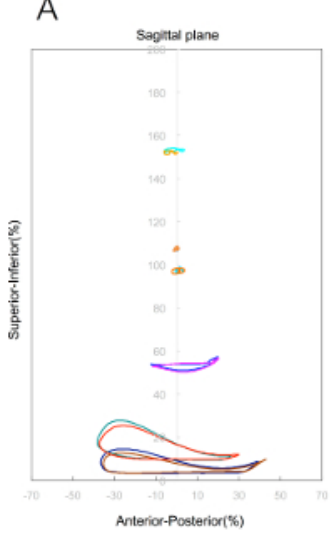

B

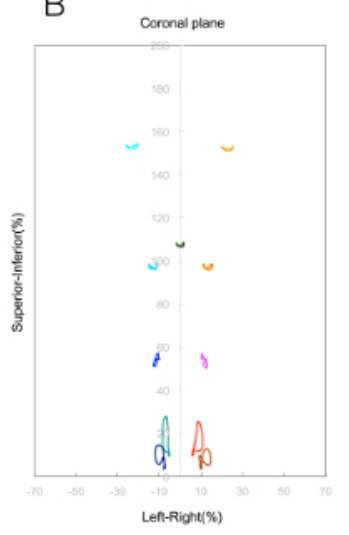

C

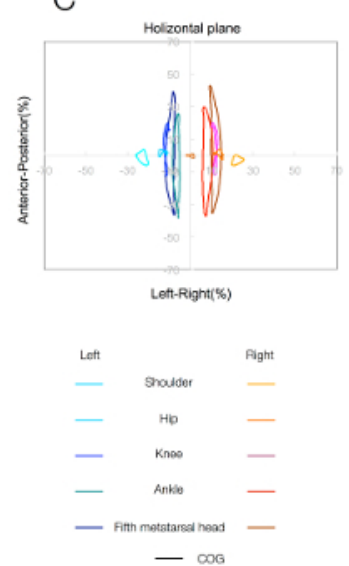

Figure 1: Lissajous overview picture (LOP) consisting of three figures: (A) sagittal plane, (B) coronal plane, and (C) horizontal plane. Each trajectory shows the averaged gait-cycle movements of joint markers and hypothesized center of gravity. Please click here to view a larger version of this figure. 
A

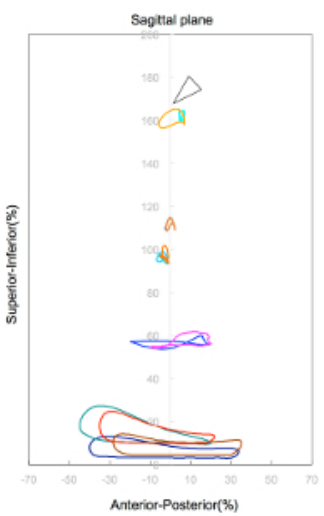

B

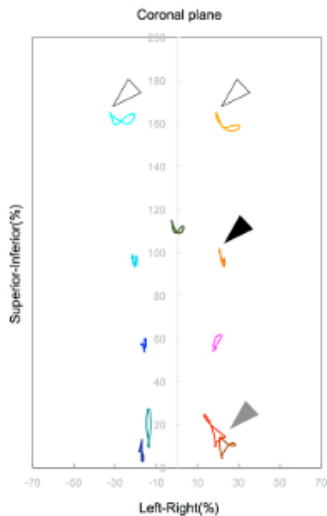

C

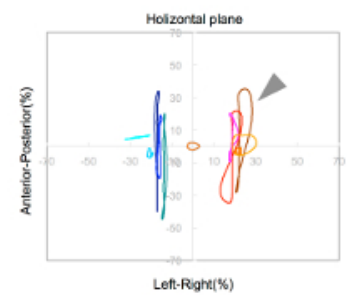

Figure 2: Lissajous overview picture (LOP) of a representative stroke patient: (A) sagittal plane, (B) coronal plane, and (C) horizontal plane. Increased trunk movement (white arrow), hip hiking (black arrow), and circumduction (gray arrow) were observed from a holistic overview using LOP. Please click here to view a larger version of this figure.

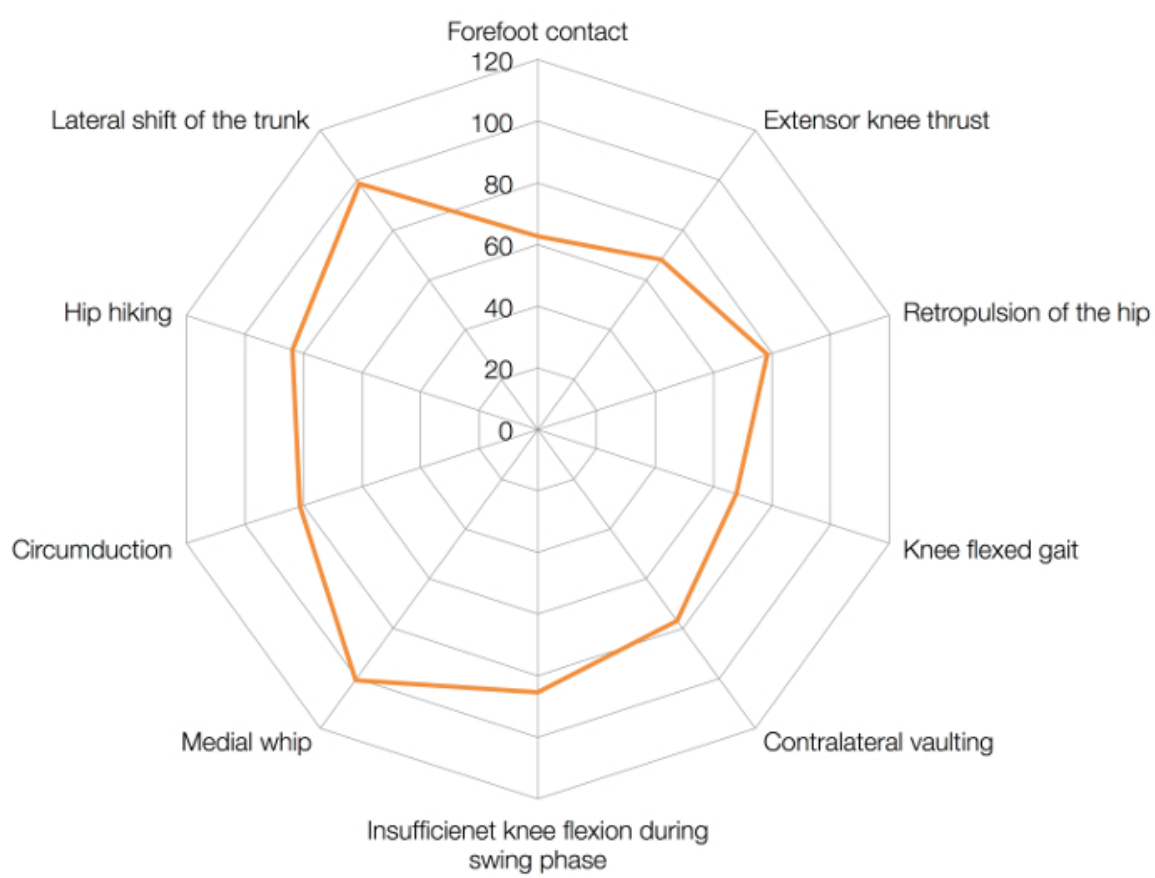

Figure 3: Radar chart of abnormal gait indices. The average score of healthy subjects is set to 50 . A high standard score represents high abnormality. Please click here to view a larger version of this figure.

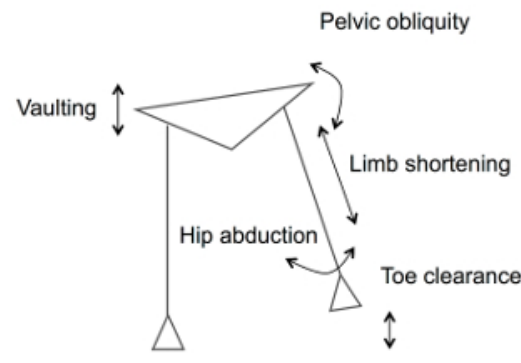

\section{B}

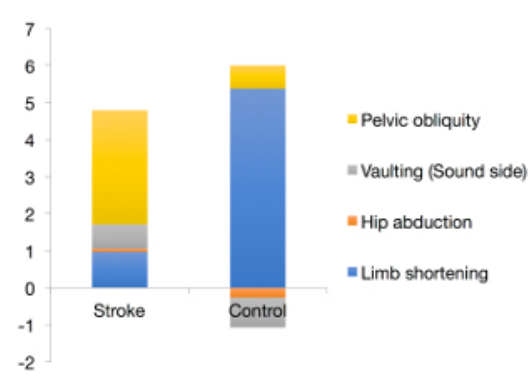

Figure 4: Graph showing toe clearance strategies in a stroke patient vs. a healthy subject. Toe clearance is achieved solely by limb shortening in the healthy subject, whereas compensatory movements (hip hiking, hip abduction, and vaulting) also affect toe clearance in the stroke patient. Please click here to view a larger version of this figure. 


\begin{tabular}{|c|c|}
\hline Gait patterns & Formula \\
\hline Hip hiking & $\begin{array}{l}\text { the difference between the maximum value of the } Z \text { coordinate of the hip joint marker during } \\
\text { the swing phase and the } Z \text { coordinate of the contralateral hip joint marker at the same time, } \\
\text { corrected for the mean left-right difference of the } Z \text { coordinate during the double support phase }\end{array}$ \\
\hline Circumduction & $\begin{array}{l}\text { the difference in distance between the lateral-most } X \text { coordinate of the ankle joint marker during } \\
25-75 \% \text { of the swing phase and the medial-most X coordinate during } 25-75 \% \text { of the stance } \\
\text { phase }\end{array}$ \\
\hline Forefoot contact & $\begin{array}{l}\text { the difference in distance between the } \mathrm{Z} \text { coordinate of the ankle joint marker and the } \mathrm{Z} \\
\text { coordinate of the toe marker at initial contact, minus the difference in distance between the } Z \\
\text { coordinates of the ankle joint marker and toe marker during standing }\end{array}$ \\
\hline Retropulsion of the hip & $\begin{array}{l}\text { the average distance between the } Y \text { coordinate of the ankle joint and the } Y \text { coordinate of the hip } \\
\text { joint in the single stance phase }\end{array}$ \\
\hline Excessive hip external rotation & $\begin{array}{l}\text { the average distance between the } X \text { coordinate of the ankle joint and the } X \text { coordinate of the toe } \\
\text { in the swing phase }\end{array}$ \\
\hline $\begin{array}{l}\text { Excessive lateral shift of } \\
\text { the trunk over the unaffected side }\end{array}$ & $\begin{array}{l}\text { the average distance between } \\
\text { (1) the lateral most } X \text { coordinate of the midpoint between the bilateral acromions in the part of the } \\
\text { double stance phase in which the affected leg is located behind the unaffected leg and the swing } \\
\text { phase of affected leg and } \\
\text { (2) the average } X \text { coordinate of the midpoint between the bilateral ankle joints in the part of the } \\
\text { double stance phase in which the affected leg is located behind the unaffected leg }\end{array}$ \\
\hline Knee extensor thrust & $\begin{array}{l}\text { the difference between the maximum Y coordinate velocity of the knee in the single stance } \\
\text { phase of the affected leg and the treadmill gait speed }\end{array}$ \\
\hline Flexed-knee gait & the maximum knee extension angle in the single stance phase of the affected leg \\
\hline Insufficient knee flexion during the swing phase & $\begin{array}{l}\text { the maximum knee flexion angle in the swing phase compared to the angle of knee flexion for } \\
\text { the healthy subjects }\end{array}$ \\
\hline \multirow[t]{2}{*}{ Medial whip } & $\begin{array}{l}\text { the distance between the lateral-most } X \text { coordinate of the ankle joint during } 75-100 \% \text { of the } \\
\text { stance phase and the medial-most X coordinate of the ankle joint during } 25-75 \% \text { in the stance } \\
\text { phase of the affected leg }\end{array}$ \\
\hline & $\mathrm{X}, \mathrm{Y}$, and $\mathrm{Z}$ coordinate indicates lateromedial, anteroposteriol, and vertical, respectively \\
\hline
\end{tabular}

Table 1: Formulas for abnormal gait patterns

\section{Discussion}

In the current report, a simplified 3DGA method was proposed. This system was designed to be used in daily practice and involves a simplified measurement method and clinician-friendly presentation of results.

Several previous reports have used 3DGA and a basic knowledge of human gait has been previously established ${ }^{21}$. 3DGA has the potential to contribute to rehabilitation clinics, for example, by improving the understanding of the pathology of gait disorders, for use in treatment planning, or in monitoring intervention effects. However, the use of 3DGA in rehabilitation clinics remains fairly low. There are several possible barriers to the use of 3DGA in clinical settings. The time required is likely a major obstacle, as research-based 3DGA usually requires considerable preparation time (i.e., for calibration of the system and mounting of the markers). Another issue is that results interpretation can be challenging. Gait disorders usually comprise several movement abnormalities, and understanding the various graphs resulting from analysis requires experience. In terms of clinical feasibility, analysis systems with simplified methods and intuitive data presentation would be helpful.

The considerable preparation time required to perform existing analysis methods is related to the pursuit of high measurement accuracy. However, the motion speed of patients with gait disorders is limited and in daily rehabilitation practice we may not require this level of accuracy. In the current method, the protocol is simplified and can be performed in a time period that should be short enough for feasibility in daily rehabilitation practice while maintaining the requirements for evaluating patients with movement disorders ${ }^{13,15,16,17}$.

In the current method, the method of results presentation meets clinical requirements. In rehabilitation clinics, therapists generally begin by evaluating holistic gait patterns. The LOP is designed to help clinicians evaluate holistic gait patterns intuitively using a representative depiction (i.e., a figure) in which marker trajectories are placed according to actual positional relationships. The development of abnormal gait indices used herein was also based on clinical experience. Indices were developed to quantify the extent of clinically common abnormal gait patterns observed in stroke patients, and the concurrent validities of all indices have been confirmed via clinical observation by physical therapists ${ }^{13,15,16}$

In addition to being an objective evaluation method that reflects clinical experience, the proposed method will ideally contribute to the development of new rehabilitation strategies. The analysis of toe clearance strategies goes beyond conventional clinical evaluation and has the potential to contribute to the planning of targeted rehabilitation treatments. In the proposed method and analysis, clinicians are presented with two targets for rehabilitation; the extent of toe clearance and the extent of compensation to achieve it. In itself, increasing toe clearance is important for safe walking; however, reducing compensation may also improve walking efficiency ${ }^{22}$. In the current method, clinicians would have access to both sets of information as indices of safe walking and walking efficiency, which may contribute to developing targeted rehabilitation strategies to improve the safety and efficiency of walking in rehabilitation patients. 
Given the abovementioned qualities, this measurement and analysis method could facilitate the use of 3DGA in the rehabilitation clinic by providing an objective method with which to evaluate gait patterns in rehabilitation patients. Moreover, it could enable more accurate estimation of the effect of intervention than of ordinary scales used in the clinic, which could contribute to further improvement of intervention quality in the rehabilitation field.

This study was not without limitations. Considering the reduced number of markers and relatively low sampling rate, the accuracy of measurement in this system may be limited compared to existing systems. This may be a critical consideration when analyzing quick movements such as those of sports professionals. In particular, when evaluating joint angles or joint movements, the simplified marker set used in this method may be insufficient to accurately estimate joint positions. Accordingly, the analysis which emphasizes on the joint movement, such as the analysis for planning cerebral palsy surgery, could also be limited with this simplified system. Although the system was validated by the clinical evaluation, the psychometric properties of this method have not yet been defined in comparison with the gold standard method. The technical limitations should be further confirmed in future studies.

However, in patients with gait disorders, motion speed is limited and differences in performance are evident when compared with healthy subjects. Therefore, in daily practice, we may not require the level of accuracy provided by existing methods. Further, in this method, results are presented as an averaged gait pattern for a $20 \mathrm{~s}$ measurement period, which is longer than that of most conventional methods of measuring overground gait. This feature could enhance the accuracy and reliability of measurement.

To conclude, in this study, a simplified and intuitive 3DGA method was introduced. To facilitate the use of 3DGA in improving the quality of rehabilitation clinics, a clinically feasible measurement method should be developed. The clinician-friendly method introduced here may encourage further development of clinically feasible measurement models and the implementation of 3DGA in daily rehabilitation clinics.

\section{Disclosures}

The authors have nothing to disclose.

\section{Acknowledgements}

This study was funded by Fujita Health University [Grant Number 2015100341].

\section{References}

1. Chen, G., Patten, C., Kothari, D.H., Zajac, F.E. Gait differences between individuals with post-stroke hemiparesis and non-disabled controls at matched speeds. Gait Posture. 22 (1), 51-6 (2005).

2. Stanhope, V.A., Knarr, B.A., Reisman, D.S., Higginson, J.S. Frontal plane compensatory strategies associated with self-selected walking speed in individuals post-stroke. Clin Biomech. 29 (5), 518-22 (2014).

3. Nadeau, S., Betschart, M., Bethoux, F. Gait analysis for poststroke rehabilitation: the relevance of biomechanical analysis and the impact of gait speed. Phys Med Rehabil Clin N Am. 24 (2), 265-76 (2013).

4. Balaban, B., Tok, F. Gait disturbances in patients with stroke. PM\& R. 6 (7), 635-42 (2014).

5. Roche, N., Pradon, D., Cosson, J., Robertson, J. Categorization of gait patterns in adults with cerebral palsy: a clustering approach. Gait Posture. 39 (1), 235-40 (2014).

6. Baker, R., Esquenazi, A., Benedetti, M.G., Desloovere, K. Gait analysis: clinical facts. Eur J Phys Rehabil Med. 52 (4), $560-74$ (2016).

7. Nadeau, S., Duclos, C., Bouyer, L., Richards, C.L. Guiding task-oriented gait training after stroke or spinal cord injury by means of a biomechanical gait analysis. Prog Brain Res. 192, 161-80 (2011).

8. Wikström, J., Georgoulas, G., Moutsopoulos, T., Seferiadis, A. Intelligent data analysis of instrumented gait data in stroke patients-a systematic review. Comput Biol Med. 51, 61-72 (2014).

9. Davis, R.B., Õunpuu, S., Tyburski, D., Gage, J.R. A gait analysis data collection and reduction technique. Hum Mov Sci. 10 (5), 575-87 (1991).

10. Cappozzo, A., Catani, F., Della Croce, U., Leardini, A. Position and orientation in space of bones during movement: anatomical frame definition and determination. Clin Biomech. 10 (4), 171-8 (1995).

11. Leardini, A., Biagi, F., Merlo, A., Belvedere, C., Benedetti, M.G. Multi-segment trunk kinematics during locomotion and elementary exercises. Clin Biomech. 26 (6), 562-71 (2011).

12. Cimolin, V., Galli, M. Summary measures for clinical gait analysis: a literature review. Gait posture. 39 (4), 1005-10 (2014).

13. Itoh, N., et al. Quantitative assessment of circumduction, hip hiking, and forefoot contact gait using Lissajous figures. Japanese J Compr Rehabil Sci. 3, 78-84 (2012)

14. Ohtsuka, K., et al. Application of Lissajous overview picture in treadmill gait analysis. Japanese J Compr Rehabil Sci. 6, 33-42 (2015).

15. Mukaino, M., et al. Feasibility of a Simplified, Clinically Oriented, Three-dimensional Gait Analysis System for the Gait Evaluation of Stroke Patients. Prog Rehabil Med. 1, 20160001 (2016).

16. Tanikawa, H., Ohtsuka, K., Mukaino, M., Inagaki, K., Matsuda, F., Teranishi, T. et al. Quantitative assessment of retropulsion of the hip, excessive hip external rotation, and excessive lateral shift of the trunk over the unaffected side in hemiplegia using three-dimensional treadmill gait analysis. Top Stroke Rehabil. 23(5), 311-17 (2016).

17. Hishikawa, N., Tanikawa, H., Ohtsuka, K., Mukaino, M., Inagaki, K., Matsuda, F. et al. Quantitative assessment of knee extensor thrust, flexed-knee gait, insufficient knee flexion during the swing phase, and medial whip in hemiplegia using three-dimensional treadmill gait analysis. Top Stroke Rehabil. in press.

18. Perry, J. The mechanics of walking in hemiplegia. Clin Orthop Relat Res. 63, 23-31 (1969).

19. Matsuda, F., Mukaino, M., Ohtsuka, K., Tanikawa, H., Tsuchiyama, K., Teranishi, T. et al. Biomechanical factors behind toe clearance during the swing phase in hemiparetic patients. Top Stroke Rehabil. 24(3), 177-82 (2016). 
20. Matsuda, F., Mukaino, M., Ohtsuka, K. Analysis of strategies used by hemiplegic stroke patients to achieve toe clearance. Japanese $J$ Compr Rehabil Sci. 7, 111-8 (2015).

21. Perry, J., Burnfield, J.M. Gait analysis : normal and pathological function. 1st ed. Slack incorporated. New Jersey. (1992).

22. Olney, S., Monga, T., Costigan, P. Mechanical energy of walking of stroke patients. Arch Phys Med Rehabil. 67(2), 92-98 (1986). 